21

\title{
Directed evolution of thermotolerant malic enzyme for improved malate production
}

(1)

Running title: Directed evolution of thermotolerant malic enzyme

8

Yumi Morimoto ${ }^{\text {a }}$, Kohsuke Honda ${ }^{\text {a,b, }}{ }^{*}$, Xiaoting Ye ${ }^{\text {a }}$, Kenji Okano ${ }^{\text {a }}$, and

Hisao Ohtake ${ }^{\text {a }}$

(1)

${ }^{a}$ Department of Biotechnology, Graduate School of Engineering, Osaka University, 2-1 Yamadaoka, Suita, Osaka 565-0871, Japan ${ }^{b}$ PRESTO, Japan Science and Technology Agency (JST), 4-1-8 Honcho Kawaguchi, 5

$$
\text { Saitama 332-0012, Japan }
$$

6

17

$19{ }^{*}$ Corresponding author. Tel: +81 66879 7438; Fax: +81 668797439

20 E-mail address: honda@bio.eng.osaka-u.ac.jp (K. Honda) 
23 The directed evolution of the thermotolerant NADP(H)-dependent malic enzyme from

24 Thermococcus kodakarensis was conducted to alter the cofactor preference of the 25 enzyme from $\mathrm{NADP}(\mathrm{H})$ to $\mathrm{NAD}(\mathrm{H})$. The construction and screening of two 26 generations of mutant libraries led to the isolation of a triple mutant that exhibited 27 6-fold higher $k_{\mathrm{cat}} / K_{\mathrm{m}}$ with $\mathrm{NAD}^{+}$than the wild type. We serendipitously found that, in 28 addition to the change in the cofactor preference, the reaction specificity of the mutant 29 enzyme was altered. The reductive carboxylation of pyruvate to malate catalyzed by 30 the wild type enzyme is accompanied by $\mathrm{HCO}_{3}{ }^{-}$-independent reduction of pyruvate and 31 gives lactate as a byproduct. The reaction specificity of the triple mutant was significantly shifted to malate production and the mutant gave a less amount of the byproduct than the wild type. When the triple mutant enzyme was used as a catalyst for pyruvate carboxylation with $\mathrm{NADH}$, the enzyme gave 1.2 times higher concentration of malate than the wild type with NADPH. Single-point mutation analysis revealed that the substitution of Arg221 with Gly is responsible for the shift in reaction specificity. This finding may shed light on the catalytic mechanisms of malic enzymes and other related $\mathrm{CO}_{2}$ - and/or $\mathrm{HCO}_{3}{ }^{-}$-fixing enzymes.

40 Key words: malic enzyme; directed evolution; cofactor preference; reaction specificity; 
$41 \quad \mathrm{HCO}_{3}{ }^{-}$fixation

42 
43 Thermophilic enzymes have been receiving increased attention as a promising tool for

44 industrial biotechnology owing to their high operational stability, co-solvent

45 compatibility, and low risk of contamination (1-3). Recently, we have developed a

46 simple approach to design and construct an in vitro artificial metabolic pathway using

47 thermophilic enzymes (4-6). In this approach, recombinant mesophiles (e.g.,

48 Escherichia coli) having heterologous thermophilic enzymes are used as biocatalytic

49 modules to construct an in vitro pathway. The heat-treatment of recombinant cells

50 results in the denaturation of indigenous enzymes and the elimination of undesired side

51 reactions; therefore, biocatalytic modules that are as highly selective as purified enzymes can be prepared in one step. By rationally combining these modules, an in vitro synthetic pathway specialized for chemical manufacture can be constructed. Previously, we constructed a chimeric Embden-Meyerhof (EM) pathway using nine recombinant E. coli strains, each of which overproduces a thermophilic glycolytic enzyme (5). In this pathway, an enzyme couple of a phosphate-dependent glyceraldehyde-3-phosphate dehydrogenase (GAPDH) and an ATP-generating

58 phosphoglycerate kinase (PGK) of a bacterial/eukaryotic EM pathway was substituted with a non-phosphorylating GAPDH (GAPN) of the archaeal modified EM pathway.

60 Consequently, the chimeric pathway with balanced ATP/ADP consumption and 
regeneration rates could be constructed. carboxylation of pyruvate to malate. Ohno et al. reported the fixation of $\mathrm{HCO}_{3}{ }^{-}$to pyruvate using the malic enzyme from Pseudomonas diminuta coupled with glucose 6-phosphate dehydrogenase for NADH regeneration (7). The system is capable of converting $100 \mathrm{mM}$ pyruvate to $38 \mathrm{mM}$ malate within $24 \mathrm{~h}$. A similar experiment using the Brevundimonas diminuta malic enzyme coupled with an electrode-mediated $\mathrm{NADH}$ regeneration system has resulted in a nearly $1.1 \mathrm{mmol}$ of malate production from $12.5 \mathrm{mmol}$ of pyruvate and $2.5 \mathrm{mmol}$ of $\mathrm{HCO}_{3}{ }^{-}$(8). We have demonstrated that the integration of the thermotolerant NADPH-dependent malic enzyme from Thermococcus kodakarensis (TkME) (9) to the chimeric EM pathway enables the construction of a cofactor-balanced and $\mathrm{HCO}_{3}{ }^{-}$-fixing synthetic pathway, through which the direct conversion of glucose to malate can be achieved (6). However, the thermal degradation of the redox cofactors $\mathrm{NADP}^{+}$and $\mathrm{NADPH}$ tends to be a major obstacle to the long-term operation of the in vitro metabolic system because, unlike living biological systems, it is not equipped with the complete enzyme apparatus for the de novo synthesis of these cofactors. The decomposition of the redox cofactors leads to the decline in the catalytic abilities of GAPN and TkME. Consequently, the 
conversion rate through the synthetic pathway gradually decreases. Furthermore, we found that the TkME-mediated malate production is accompanied by $\mathrm{HCO}_{3}{ }^{-}$-independent reduction of pyruvate to lactate (6). Owing to these facts, the final malate concentration is only modest $(2.6 \mathrm{mM})$ and lactate was produced as the byproduct (6).

One of the possible approaches to achieve a long-term operation of the in vitro system and to obtain a higher titer of malate is to alter the cofactor preference of the NADPH-dependent malic enzyme from $\mathrm{NADP}(\mathrm{H})$ to $\mathrm{NAD}(\mathrm{H})$, because the thermal stabilities of $\mathrm{NADP}^{+}$and NADPH are significantly lower than those of $\mathrm{NAD}^{+}$and NADH, particularly under neutral and alkaline conditions (10) (Figure S1). We herein report the alteration of the cofactor preference of TkME, the bottleneck enzyme in the synthetic pathway for malate production, by a directed evolution approach. We serendipitously find that, in addition to the change in the cofactor preference, the reaction specificity of the mutant enzyme is significantly shifted to malate production. MATERIALS AND METHODS Plasmid construction Escherichia coli JM109 was used as the host strain for DNA manipulation. The gene coding for TkME was amplified by polymerase chain reaction 
97 (PCR) from the chromosomal DNA of T. kodakarensis KOD1 using a primer set of

98 ME-F and ME-R (Table 1). The amplicon was digested with SacI and HindIII and

99 inserted into the corresponding restriction sites of pUC18 (Takara Bio, Ohtsu, Japan).

100 The resulting plasmid was designated as pUC-ME. Expression vectors encoding a

101 hexahistidine (His6)-tagged TkME and mutant enzymes were constructed using 102 pET-21a (Novagen, Madison, WI, USA). Genes were PCR-amplified with a primer 103 pair of His_ME-F and His_ME-R (Table 1), digested with NdeI and HindIII, and then 104 inserted to the corresponding restriction sites of pET-21a. The resulting plasmids were 105 transformed in E. coli Rosetta2 (DE3) pLysS (Novagen). The site-directed 106 mutagenesis of TkME was conducted using a PrimeSTAR mutagenesis kit (Takara Bio)

107 and adequate sets of oligonucleotide primers listed in Table 1.

109 Construction of mutant library Error-prone PCR was conducted using pUC-ME as

110 the template. GoTaq DNA polymerase (Promega, Madison, WI, USA) and M13

111 primers (Table 1) were used for the amplification according to the manufacturer's

112 instruction. Additionally, $0.1 \mathrm{mM} \mathrm{MnCl}_{2}$ was added to the PCR mixture to increase

113 error rate. Under the experimental conditions, 1-4 nucleotides of the gene were mutated on average $(n=4)$. Amplified DNAs were introduced into the SacI/HindIII 
115 restriction sites of pUC18 and then transformed in E. coli JM109 (Takara Bio).

116 Transformants harboring the correct insertion were selected through the blue-white

117 screening on a Luria-Bertani (LB) agar medium supplemented with $100 \mu \mathrm{g} \mathrm{ml}{ }^{-1}$

118 ampicillin, $100 \mu \mathrm{g} \mathrm{ml}^{-1}$ 5-bromo-4-chloro-3-indolyl- $\beta$-D-galactoside (X-gal), and $1 \mathrm{mM}$

119 isopropyl- $\beta$-D-thiogalactopyranoside (IPTG).

120

121 High-throughput screening A colorimetric high-throughput assay was employed

122 for the first-round screening of the mutant library. Transformants were cultivated in a

123 96-deep-well plate containing $500 \mu$ l per well of LB medium supplemented with $100 \mu \mathrm{g}$

$124 \mathrm{ml}^{-1}$ ampicillin and $0.2 \mathrm{mM}$ IPTG. Cells were cultivated at $37^{\circ} \mathrm{C}$ for $15 \mathrm{~h}$ with orbital

125 shaking, harvested by centrifugation $(800 \times g, 10 \mathrm{~min})$, and then resuspended in $50 \mathrm{mM}$

126 Tris- $\mathrm{HCl}(\mathrm{pH}$ 8.0). Cell concentration was adjusted to approximately 60 and $20 \mathrm{mg}$

127 wet cells $\mathrm{ml}^{-1}$ for the screening of the first- and second-generation libraries, respectively.

128 The cell suspension was incubated at $70^{\circ} \mathrm{C}$ for $30 \mathrm{~min}$ and mixed with an equal volume

129 of a mixture comprising $20 \mathrm{mM}$ L-malate, $2 \mathrm{mM} \mathrm{NAD}{ }^{+}, 10 \mathrm{mM} \mathrm{MnCl}_{2}, 12 \mu \mathrm{M}$

130 1-methoxy-5-methylphenazinium methyl sulfate (1-methoxy-PMS, Dojindo, Kumamoto,

131 Japan), $300 \mu \mathrm{M}$ 3-(4,5-dimethylthiazol-2-yl)-2,5-diphenyltetrazolium bromide (MTT,

132 Dojindo), and $50 \mathrm{mM}$ Tris- $\mathrm{HCl}$ ( $\mathrm{pH} \mathrm{8.0).} \mathrm{After} \mathrm{incubation} \mathrm{at} \mathrm{room} \mathrm{temperature} \mathrm{for} 1 \mathrm{~h}$, 
133 the accumulation of formazan crystals, which is formed by the reduction of MTT, was 134 visually examined.

136 Enzyme preparation E. coli having $\mathrm{COOH}$-terminal His6-tagged malic enzymes

137 were cultivated in a 500-ml Erlenmeyer flask containing $200 \mathrm{ml}$ of LB medium 138 supplemented with $100 \mu \mathrm{g} \mathrm{ml}^{-1}$ ampicillin and $30 \mu \mathrm{g} \mathrm{ml}^{-1}$ chloramphenicol. Cells were 139 cultivated at $37^{\circ} \mathrm{C}$ for $15 \mathrm{~h}$ with orbital shaking. Gene expression was induced by 140 adding $0.2 \mathrm{mM}$ IPTG at the late log phase. Cells were harvested by centrifugation, 141 resuspended in $50 \mathrm{mM}$ Tris- $\mathrm{HCl}(\mathrm{pH}$ 8.0) containing $20 \mathrm{mM}$ imidazole and $500 \mathrm{mM}$ $142 \mathrm{NaCl}$, and then disrupted with a UD-201 ultrasonicator (Kubota, Osaka, Japan). The 143 crude lysate was heated at $70^{\circ} \mathrm{C}$ for $30 \mathrm{~min}$. Cell debris and denatured proteins were 144 removed by centrifugation at $12,000 \times g$ and $4{ }^{\circ} \mathrm{C}$ for $10 \mathrm{~min}$. The enzymes were 145 homogenously purified by eluting the heat-treated lysates of recombinant cells through 146 a HisTrap HP column (GE Healthcare, Piscataway, NJ) with a linear gradient of 147 imidazol. The purified enzymes were dialyzed against $50 \mathrm{mM}$ Tris-HCl ( $\mathrm{pH} \mathrm{8.0).}$

148 Protein concentration was measured with the Bio-Rad assay system (Bio-Rad, Hercules, 149 CA, USA) using bovine serum albumin as the standard. 
152 determined by monitoring the concomitant reduction of $\mathrm{NAD}^{+}$. The standard reaction

153 mixture was composed of $50 \mathrm{mM}$ Tris- $\mathrm{HCl}$ (pH 8.0), $5 \mathrm{mM} \mathrm{MnCl}_{2}, 5 \mathrm{mM} \mathrm{NAD}^{+}$, and 154 an appropriate amount of enzyme. Alternatively, $0.5 \mathrm{mM} \mathrm{NADP}^{+}$was used instead of

$155 \mathrm{NAD}^{+}$when needed. The mixture was preincubated at $70^{\circ} \mathrm{C}$ for $2 \mathrm{~min}$, and then the 156 reaction was initiated by adding L-malate at a final concentration of $30 \mathrm{mM}$. The 157 absorbance at $340 \mathrm{~nm}$ was monitored using a UV-VIS spectrophotometer (Model 158 UV-2450, Shimadzu, Kyoto, Japan). Molar extinction coefficients of 6200 and 6300 $159 \mathrm{M}^{-1} \mathrm{~cm}^{-1}$ for NADH and NADPH, respectively, were used to calculate the reaction rate. Malate production The reaction mixture $(1 \mathrm{ml})$ contained $500 \mathrm{mM}$ HEPES-NaOH (pH 7.0), $5 \mathrm{mM} \mathrm{NH} \mathrm{NH}_{4} \mathrm{Cl} 0.5 \mathrm{mM} \mathrm{MnCl}_{2}, 30 \mathrm{mM}$ pyruvate, $30 \mathrm{mM}$ glucose, $85 \mathrm{mM}$ $\mathrm{NaHCO}_{3}, 2 \mu \mathrm{g}$ of TkME, $2 \mathrm{U}$ of glucose 1-dehydrogenase (GDH, Thermostable 164 Enzyme Laboratory, Kobe, Japan), and $1 \mathrm{mM}$ NADH or NADPH. The reaction mixture was stirred in a sealed container $(\phi 15 \times 50 \mathrm{~mm})$ kept at $50^{\circ} \mathrm{C}$, and the headspace

166 of the container was equilibrated with $\mathrm{CO}_{2}$ gas for 5 min before the addition of the substrates (pyruvate and glucose). After the reaction at $50^{\circ} \mathrm{C}$, the mixture was 168 ultrafiltered using Amicon 3K (Millipore, Bedford, MA) and subjected to 
high-performance liquid chromatography (HPLC), performed as described elsewhere (6).

RESULTS

173 Screening of mutant library In total, 1016 transformants were screened with the

174 MTT colorimetric assay, and the positive clones were further evaluated by determining

175 their malate decarboxylating activities under standard assay conditions. Consequently,

17616 transformants were found to exhibit higher specific activities with NAD ${ }^{+}$than that of

177 the transformant having the wild type TkME (data not shown). Among them, the

178 transformant exhibiting the highest activity with $\mathrm{NAD}^{+}$was selected for further study.

179 Sequence analysis of the encoding gene revealed that there were three substitutions at

180 nucleotide positions of $661(\underline{A G G} \rightarrow \underline{G G G}$, resulting in an amino acid substitution of

$181 \mathrm{R} 221 \mathrm{G}), 683(\mathrm{~A} \underline{\mathrm{A} G} \rightarrow \mathrm{A} \underline{\mathrm{GG}}$, resulting in K228R), and 1179 (GTㅡㅡ $\rightarrow \mathrm{GTT}$, resulting in a synonymous mutation of V393V). The plasmid encoding the R221G/K228R mutant of TkME was then used as the template for the second round of error-prone PCR.

184 After screening the second-generation library, which consisted of 1384 mutants, the triple mutant having an additional amino acid substitutions of I310V, which is caused 
187 highest activity with $\mathrm{NAD}^{+}$. For the determination of the specific activities of TkME

188 and the mutant enzymes, these enzymes were fused with a His6 tag on their $\mathrm{COOH}$

189 terminals and homogeneously purified by Ni-affinity column chromatography. The

190 specific malate decarboxylating activities of R221G/K228R and R221G/K228R/I310V

191 enzymes with $\mathrm{NAD}^{+}$were 1.6 and 3.9 times higher than that of the wild type,

192 respectively (Table 2). No significant change was observed in the thermal stability of

193 the wild type and mutant enzymes (data not shown).

194

195 Kinetic analysis Since both the oxidative carboxylation of pyruvate (pyruvate +

$196 \mathrm{HCO}_{3}{ }^{-}+\mathrm{NAD}(\mathrm{P}) \mathrm{H}+2 \mathrm{H}^{+} \rightarrow$ malate $+\mathrm{NAD}(\mathrm{P})^{+}+\mathrm{H}_{2} \mathrm{O} ; \Delta \mathrm{G}^{0^{\prime}}=+7.3 \mathrm{~kJ} \mathrm{~mol}^{-1}$ ) and the

197 lactate oxidation (lactate $+\mathrm{NAD}^{+} \rightarrow$ pyruvate $+\mathrm{NADH}+\mathrm{H}^{+} ; \Delta \mathrm{G}^{0^{\prime}}=+14.8 \mathrm{~kJ} \mathrm{~mol}^{-1}$ )

198 catalyzed by TkME are thermodynamically unfavorable $(11,12)$, these reactions hardly

199 proceed under the standard assay conditions. Therefore, the kinetic parameters of the

200 enzymes were obtained from their specific malate decarboxylating activities using

201 various concentrations of $\mathrm{NAD}^{+}$and $\mathrm{NADP}^{+}$(Table 2). Although the $K_{\mathrm{m}}$ of the

202 R221G/K228R mutant for $\mathrm{NAD}^{+}$was less than half that of the wild type, that of the

203 mutant for $\mathrm{NADP}^{+}$was 30 times higher than that of the wild type. Similarly, more

204 than 1.5-fold increase was observed in the $k_{\text {cat }}$ of the mutant with $\mathrm{NAD}^{+}$although that 
205 with $\mathrm{NADP}^{+}$was markedly lowered. These facts indicate that the increased specific 206 activity of the R221G/K228R mutant with $\mathrm{NAD}^{+}$was attributed to the shift in the 207 cofactor preference of the enzyme to $\mathrm{NAD}^{+}$. By contrast, the increased specific 208 activity of the triple mutant (R221G/K228R/I310V) was more likely due to the 209 improvement in the catalytic ability of the enzyme rather than to the shift in the cofactor 210 preference. In fact, although the R221G/K228R mutant exhibited a significantly lower 211 specific activity with $\mathrm{NADP}^{+}$than the wild type, the additional mutation of I310V 212 restored the specific activity regardless of the cofactor used.

213 To further investigate the impact of each mutation (R221G, K228R, or I310V) on 214 cofactor preference, site-directed mutations were introduced into the His6-tagged TkME.

215 Among the resulting single-point mutants, only the K228R mutant showed a 216 significantly low $K_{\mathrm{m}}$ for $\mathrm{NAD}^{+}$. The other two mutations (R221G and I310V) mainly 217 affected $k_{\text {cat }}$ but not the affinity to $\mathrm{NAD}^{+}$. Although the single-point mutation of 218 R221G had no apparent effect on the $K_{\mathrm{m}}$ for NAD ${ }^{+}$, our observation that the $K_{\mathrm{m}}$ of the 219 R221G/K228R mutant for $\mathrm{NAD}^{+}$was significantly lower than that of the K228R mutant implied that these two mutations had a synergistic effect on the affinity of the enzyme to $\mathrm{NAD}^{+}$ 
223 Malate production The malate production assay was carried out by coupling the 224 wild type or triple mutant of TkME with the thermotolerant GDH, which can accept 225 both $\mathrm{NAD}^{+}$and $\mathrm{NADP}^{+}$, to regenerate the redox cofactor. Furthermore, the oxidation 226 of glucose to gluconolactone catalyzed by GDH (glucose $+\mathrm{NAD}(\mathrm{P})^{+} \rightarrow$ gluconolactone $227+\mathrm{NAD}(\mathrm{P}) \mathrm{H}+\mathrm{H}^{+} ; \Delta \mathrm{G}^{0^{\prime}}=-4.0 \mathrm{~kJ} \mathrm{~mol}^{-1}$ ) gives a negative free energy change and can 228 shift the overall chemical equilibrium to the direction of malate production. 229 Previously, we demonstrated that TkME utilizes $\mathrm{HCO}_{3}{ }^{-}$but not dissolved $\mathrm{CO}_{2}$ as the 230 active carboxylation species for pyruvate carboxylation (6). Moreover, the reductive 231 carboxylation of pyruvate catalyzed by $T k \mathrm{ME}$ is accompanied by lactate formation $232\left(\mathrm{HCO}_{3}{ }^{-}\right.$-independent reduction of pyruvate) and carboxylation rate is dependent on $233 \mathrm{HCO}_{3}{ }^{-}$concentration in the solution phase (6). At certain temperatures, $\mathrm{HCO}_{3}{ }^{-}$ 234 concentration is dependent on $\mathrm{pH}$ and the partial pressure of $\mathrm{CO}_{2}$. Thus, a relatively 235 high concentration of the buffer was employed and the headspace of the reaction vessel 236 was equilibrated with $\mathrm{CO}_{2}$ gas to maintain a certain $\mathrm{HCO}_{3}{ }^{-}$concentration.

237 When the R221G/K228R/I310V mutant was used with NADH, the mutant gave 1.2 and 2382.7 times higher malate concentration than the wild type with NADPH and NADH, 239 respectively (Fig. 1). These results can be partly explained by the alteration of the 240 cofactor preference of the mutant enzyme, since the half-life of NADH is approximately 
2411.3 times longer than that of NADPH under our experimental conditions at $50^{\circ} \mathrm{C}$ (Figure

242 S1). However, the $K_{\mathrm{m}}$ of the triple mutant for $\mathrm{NAD}^{+}$remained 190 times higher than

243 that of the wild type for $\mathrm{NADP}^{+}$. This indicates that the wild type can have a

244 comparatively higher catalytic ability at much lower NADPH concentrations than the

245 triple mutant used with NADH. On the other hand, our results demonstrated that, in

246 addition to the change in cofactor preference, the reaction specificity of the mutant

247 enzyme was significantly shifted to malate formation. Regardless of the enzyme used,

248 the malate production rate decreased time-dependently probably owing to $\mathrm{HCO}_{3}{ }^{-}$

249 consumption, whereas the lactate production rate was almost constant for $6 \mathrm{~h}$.

250 Consequently, when the wild type was used, the final lactate concentration was higher

251 than the final malate concentration. By contrast, malate was mainly produced when

252 the R221G/K228R/I310V mutant was employed as the catalyst. The total

253 concentration of malate and lactate produced by the wild type with NADPH was 1.3

254 times higher than that by the triple mutant with NADH, implying that the improved

255 malate production by the triple mutant was more likely attributed to the redirection of

256 the reaction specificity rather than to the alteration of cofactor preference. When the

R221G/K228R/I310V mutant was used with NADPH, the mutant gave lower 
total product concentration (malate + lactate) remained higher than that of lactate (Fig.

1C). The effect of each mutation point on the reaction specificity of TkME was further

261 investigated using the single-point mutants. The results showed that K228R (ratio of

malate in overall yield: 58\%) and I310V (61\%) mutants gave almost the same levels of

263 malate as the wild type (66\%) while the reaction specificity of R221G (78\%) was

264 significantly shifted to the direction of malate production. The triple mutant gave

265 slightly higher ratio of malate (86\%) than R221G, implying that the other two mutations

of K228R and I310V might have a synergistic effect on the reaction specificity of

TkME (Fig. 2).

\section{DISCUSSION}

The X-ray structural analysis of the pigeon (Columba livia) cytosolic malic enzyme

In CIME, the substitution of Lys362 with Ala leads to a 70-fold increase in the apparent

$K_{\mathrm{m}}$ for $\mathrm{NADP}^{+}$, without significantly changing the affinity of the enzyme to malate and

$\mathrm{Mn}^{2+}$ (14). Similarly, the substitution of Lys362 of the human cytosolic malic enzyme 
277 decrease in that for $\mathrm{NAD}^{+}(15)$. Amino acid sequence alignment analysis showed that

278 these Lys residues are highly conserved among TkME, ClME, and other

279 NADPH-dependent malic enzymes (Fig. 3), in spite of the fact that the similarity

280 between the whole amino acid sequences of TkME and the eukaryotic malic enzymes is

281 modest $(<20 \%)$. In this study, we confirmed that the substitution of the Lys228 of

282 TkME, which corresponds to the Lys362 of ClME, also led to a shift in cofactor

283 preference (Table 2). These observations strongly indicate that these Lys residues play

284 a crucial role in the interaction of malic enzymes with NADP(H).

285 Regardless of the cofactor used $\left(\mathrm{NADP}^{+}\right.$or $\left.\mathrm{NAD}^{+}\right)$, the substitution of the Arg221 of

286 TkME with Gly resulted in an increased $k_{\text {cat }}$ of the enzyme (Table 2). This residue

287 corresponds to the Arg354 of CIME and is well conserved particularly in

288 NADP(H)-dependent malic enzymes (Fig. 3). The crystal structural analysis of ClME

289 suggested that the Arg354 forms an ion pair with Asp345 (13). These facts imply that

290 R221G mutation in TkME causes the partial disintegration of the cofactor binding site

291 and the loss of cofactor selectivity. The structural modeling analyses indicated that

292 Ile310, which was changed to Val in the triple mutant, is also located nearby the

293 putative cofactor binding pocket of TkME (Fig. 4). However, this residue is not

294 conserved in CIME and other malic enzymes with structural information in complex 
with redox cofactors, and likely is not involved in the direct interaction with the

cofactors. This assumption is also supported by the fact that increased $k_{\text {cat }} / K_{\mathrm{m}}$ of the

I310V mutant was mainly attributed to the increased $k_{\text {cat }}$ (Table 2).

298

Under physiological conditions, malic enzymes are involved in lipogenesis (16) and

energy production (17) through $\mathrm{NAD}(\mathrm{P}) \mathrm{H}$ production concomitant with malate

decarboxylation. Therefore, most studies so far have been focused on the catalytic

mechanisms of the oxidative decarboxylation of malate to pyruvate $(18,19)$. On the

other hand, the mechanisms of malic-enzyme-mediated pyruvate carboxylation have

rarely been studied, and, to our knowledge, the key amino acid residues involved in the

interaction with $\mathrm{HCO}_{3}{ }^{-}$have not yet been identified. Recently, the utilization of carbon

dioxide as a building block for synthetic chemistry has emerged as one of the most

outstanding research topics for establishing independence from fossil reserves, and thus

the applications of $\mathrm{CO}_{2}$ - and/or $\mathrm{HCO}_{3}{ }^{-}$-fixing enzymes and microorganisms to the

production of biofuels and other useful chemicals have been intensively studied (20-23).

Our serendipitous finding of the possible key residue of TkME for pyruvate

carboxylation may shed light on the catalytic mechanisms of malic enzymes and other

related $\mathrm{CO}_{2}$ - and/or $\mathrm{HCO}_{3}{ }^{-}$-fixing enzymes. 


\section{ACKNOWLEDGMENTS}

314 We thank E. Tannous for supporting the modeling analysis. This work was supported

315 in part by the Japan Science and Technology Agency (JST), PRESTO program.

$317 \quad$ References

318 1. Persidis, A.: Extremophiles. Nat. Biotechnol., 16, 593-594 (1998).

319 2. Cava, F., Hidalgo, A. and Berenguer, J.: Thermus thermophilus as biological 320 model. Extremophiles, 13, 213-231 (2009).

3. Turner, P., Mamo, G. and Karlsson, E.N.: Potential and utilization of (2007).

4. Honda, K., Maya, S., Omasa, T., Hirota, R., Kuroda, A. and Ohtake, H.:. Production of 2-deoxyribose 5-phosphate from fructose to demonstrate a potential 204-207 (2010).

5. Ye, X., Honda, K., Sakai, T., Okano, K., Omasa, T., Hirota, R., Kuroda, A. of artificial bio-synthetic pathway using thermophilic enzymes. J. Biotechnol., 148, and Ohtake, H.: Synthetic metabolic engineering -a novel, simple technology for designing a chimeric metabolic pathway. Microb. Cell. Fact., 11:120 (2012). 
6. Ye, X., Honda, K., Morimoto, Y., Okano, K. and Ohtake, H.: Direct conversion (2013).

7. Ohno, Y., Nakamori, T., Zheng, H. and Suye, S.: Reverse reaction of malic enzyme for $\mathrm{HCO}_{3}{ }^{-}$fixation into pyruvic acid to synthesize L-malic acid with enzymatic coenzyme regeneration. Biosci. Biotechnol. Biochem., 72, 1278-1282 (2008).

8. Zheng, H., Ohno, T., Nakamori, T. and Suye, S.: Production of l-malic acid with

9. Fukuda, W., Ismail, Y.S., Fukui, T., Atomi, H. and Imanaka, T.:

10. Wu, J.T., Wu, L.H. and Knight, J.A.: Stability of NADPH: effect of various factors on the kinetics of degradation. Clin. Chem., 32,314-319 (1986).

11. Goldberg, R.N., Tewari, Y.B., Bell, D., Fazio, K. and Anderson, E.: Thermodynamics of enzyme-catalyzed reactions: Part 1. Oxidoreductases. J. Phys. 
Chem. Ref. Data, 22, 515-579 (1993).

12. Garrett, R.H. and Grisham, C.M.: Biochemistry, Saunders College Publishing, Harcourt Brace College Publishers, Orlando, (1998).

13. Yang, Z., Zhang, H., Hung, H., Kuo C., Tsai, L., Yuan H.S., Chou W., Chang, 
19. Hsu, R.Y., Mildvan, A.S., Chang, G. and Fung, C.: Mechanism of malic enzyme from pigeon liver. Magnetic resonance and kinetic studies of the role of $\mathrm{Mn}^{2+} . \mathrm{J}$. Biol. Chem., 251, 6574-6538 (1976).

20. Rosgaard, L., de Porcellinis, A.J., Jacobsen,J.H., Frigaard, N-U. and Sakuragi, Y.: Bioengineering of carbon fixation, biofuels, and biochemicals in cyanobacteria and plants. J. Biotechnol., 162, 134-147 (2012).

21. Li, H., Opgenorth, P.H., Wenick, D.G., Rogers, S., Wu, T, Higashide, W., Malati, P., Huo, Y., Cho, K. M. and Liao, J.C.: Integrated electromicrobial conversion of $\mathrm{CO}_{2}$ to higher alcohol. Science, 335, 1596 (2012).

22. Oliver, J.W.K., Machado, I.M.P., Yoneda, H. and Atsumi, S.: Cyanobacterial conversion of carbon dioxide to 2,3-butanediol. Proc. Natl. Acad. Sci. USA., 110, 1249-1254 (2013).

23. Keller, M.W., Schut, G.J., Lipscomb, G.L., Menon, A.L., Iwuchukwu, I.J., Leuko, T.T., Thorgersen, M.P., Nixon, W.J., Hawkins, A.S., Kelly, R.M., and Adams, M.W.W.: Exploiting microbial hyperthermophilicity to produce an industrial chemical, using hydrogen and carbon dioxide. Proc. Natl. Acad. Sci. USA., 110, 5840-5845 (2013). 
Legends of Figures

386

FIG. 1 Malate productions by the wild type (A, B) and R221G/K228R/I310V mutant

(C, D). Reactions were performed using either NADPH (A, C) or NADH (B, D) as a

redox cofactor. After reaction for the indicated time periods, the concentrations of malate (closed square) and lactate (open square) were determined by HPLC.

FIG. 2 Reaction specificities of the wild type and mutant enzymes. Reactions were performed with $2 \mu \mathrm{g}$ each of the enzymes using NADH as the cofactor. After the reaction for $1 \mathrm{~h}$, the ratios of malate (black bar) and lactate (gray bar) to the total product concentration (malate and lactate) were determined. The total product concentrations are shown in parenthesis.

FIG. 3 Alignment of amino acid sequences of malic enzymes near cofactor binding site. Sequences were aligned with the Genetyx program (ver. 11, GENETYX Co., Tokyo, Japan). Mutation points identified in this study are indicated by black stars. 

panel) and R221G/K228R/I310V mutant (lower panel). The model was built by

404 SWISS-MODEL-automated protein homology-modeling server (Swiss Institute of 405 Bioinformatics) using the structure of malic enzyme derived from Pyrococcus 406 horikoshii OT3 (pdb: 1WW8) as a template. These proteins share the amino acid 407 sequence identity of $61 \%$. The model was viewed and edited with PyMOL 408 (www.pymol.org). The mutated residues in the triple mutant (green) and the 409 corresponding residues in the wild type (orange) are shown as stick models, in which 410 the nitrogen atoms are blue-colored. 
TABLE 1 Oligonucleotide primers used in this study

\begin{tabular}{|c|c|c|}
\hline Primer & Sequence $\left(5^{\prime} \rightarrow 3^{\prime}\right)$ & Purpose \\
\hline ME-F & TTGAGCTC ${ }_{a}$ GAATCCCCTCGAATTCCATAGGGACAAC & \\
\hline ME-R & TTAAGCTT $\underline{b} \underline{\underline{B}}$ CTAGGGGGAACTCCСТCTACCG & Construction of pUC-ME \\
\hline His_ME-F & TTCATATG $_{\underline{c}}$ AATCCCCTCGAATTCCATAGGGA & Construction of the expression vectors encoding \\
\hline His_ME-R & TTAAGCTT $\underline{b} \underline{\underline{A} G G G G A A C T C C C T C T A C C G C T G ~}$ & His6-tagged malic enzymes \\
\hline M13-F & GTAAAACGACGGCCAGT & \\
\hline M13-R & CAGGAAACAGCTATGAC & Error-prone PCR \\
\hline R221G-F & GAGGGCGGCGAGGACCTCAACCCGTAC & Construction of the expression vector encoding \\
\hline R221G-R & GTCCTCGCCGCCCTCGTGTATTATCCC & R221G mutant \\
\hline K228R-F & AACCAGGTGAACAACGTGCTCGGCTTT & Construction of the expression vector encoding \\
\hline K228R-R & TTCGCGACGGTACGGGTTGAGGTCCTC & K228R mutant \\
\hline I310V-F & AACCAGGTGAACAACGTGCTCGGCTTT & Construction of the expression vector encoding \\
\hline I310V-R & GTTGTTCACCTGGTTCGGGAAGTCGCT & I310V mutant \\
\hline
\end{tabular}

\footnotetext{
${ }^{\mathrm{a}}$ The SacI restriction site is underlined.
} 
$414{ }^{\mathrm{b}}$ The HindIII restriction site is underlined.

$415 \quad{ }^{\mathrm{c}}$ The NdeI restriction site is underlined. 
417 TABLE 2 Specific activities and kinetic parameters of the wild type and mutant enzymes

\begin{tabular}{|c|c|c|c|c|c|c|c|c|}
\hline \multirow[t]{2}{*}{ Enzyme } & \multicolumn{2}{|c|}{ 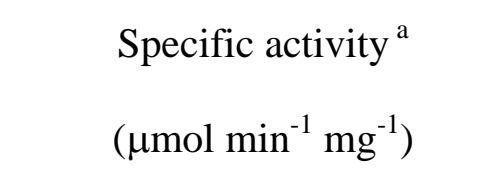 } & \multicolumn{2}{|c|}{$\begin{array}{l}K_{\mathrm{m}}^{\mathrm{b}} \\
(\mathrm{mM})\end{array}$} & \multicolumn{2}{|c|}{$\begin{array}{l}k_{\mathrm{cat}}^{\mathrm{b}} \\
\left(\mathrm{s}^{-1}\right)\end{array}$} & \multicolumn{2}{|c|}{$\begin{array}{c}k_{\mathrm{cat}} / K_{\mathrm{m}} \\
\left(\mathrm{mM}^{-1} \mathrm{~s}^{-1}\right)\end{array}$} \\
\hline & $\mathrm{NAD}^{+}$ & $\mathrm{NADP}^{+}$ & $\mathrm{NAD}^{+}$ & $\mathrm{NADP}^{+}$ & $\mathrm{NAD}^{+}$ & $\mathrm{NADP}^{+}$ & $\mathrm{NAD}^{+}$ & $\mathrm{NADP}^{+}$ \\
\hline wild type & $5.77 \pm 0.31$ & $38.4 \pm 1.2$ & $2.06 \pm 0.03$ & $0.008 \pm 0.001$ & $4.69 \pm 0.33$ & $31.1 \pm 0.59$ & 2.27 & 3760 \\
\hline R221G/K228R & $9.32 \pm 0.15$ & $4.89 \pm 0.28$ & $0.96 \pm 0.07$ & $0.244 \pm 0.007$ & $7.44 \pm 0.49$ & $6.48 \pm 0.25$ & 7.72 & 27.5 \\
\hline R221G/K228R/I310V & $22.9 \pm 0.19$ & $27.4 \pm 2.4$ & $1.54 \pm 0.09$ & $0.384 \pm 0.06$ & $21.3 \pm 0.89$ & $40.1 \pm 6.3$ & 13.8 & 104 \\
\hline R221G & $13.0 \pm 0.41$ & $52.1 \pm 3.3$ & $2.29 \pm 0.28$ & $0.025 \pm 0.007$ & $12.7 \pm 0.32$ & $42.6 \pm 2.9$ & 5.57 & 1700 \\
\hline K228R & $4.47 \pm 0.09$ & $3.57 \pm 0.08$ & $1.28 \pm 0.09$ & $0.019 \pm 0.004$ & $3.37 \pm 0.20$ & $2.97 \pm 0.20$ & 2.64 & 156 \\
\hline I310V & $22.7 \pm 0.38$ & $5.23 \pm 0.08$ & $6.55 \pm 0.25$ & $0.021 \pm 0.003$ & $37.7 \pm 1.5$ & $43.4 \pm 1.8$ & 5.76 & 2070 \\
\hline
\end{tabular}

$418{ }^{\mathrm{a}}$ Enzyme assays were performed with either $5 \mathrm{mM} \mathrm{NAD}^{+}$or $0.5 \mathrm{mM} \mathrm{NADP}^{+}$.

$419{ }^{\mathrm{b}}$ The kinetic parameters were obtained by Lineweaver-Burk analysis. 

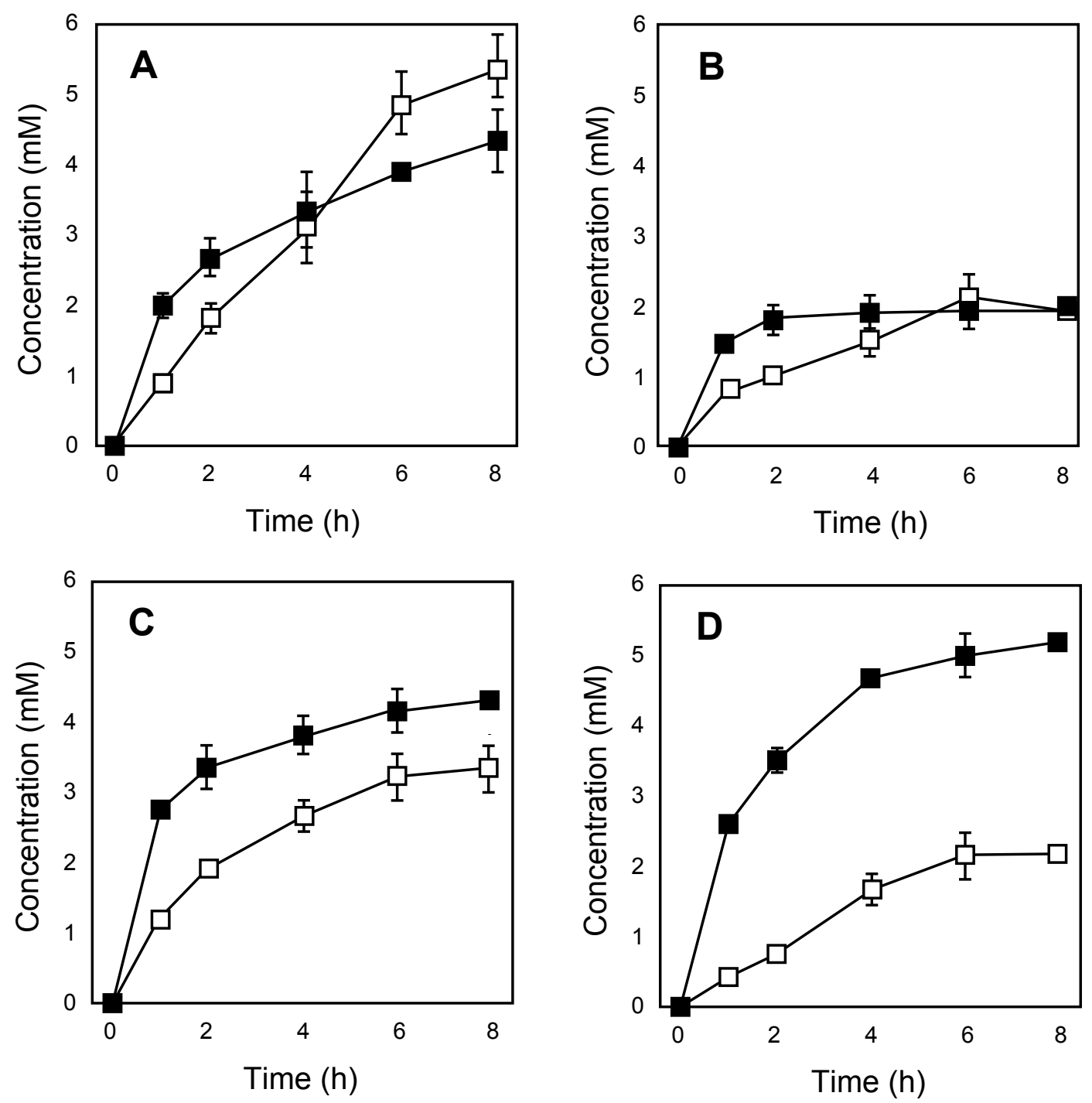

Morimoto et al., FIG. 1 


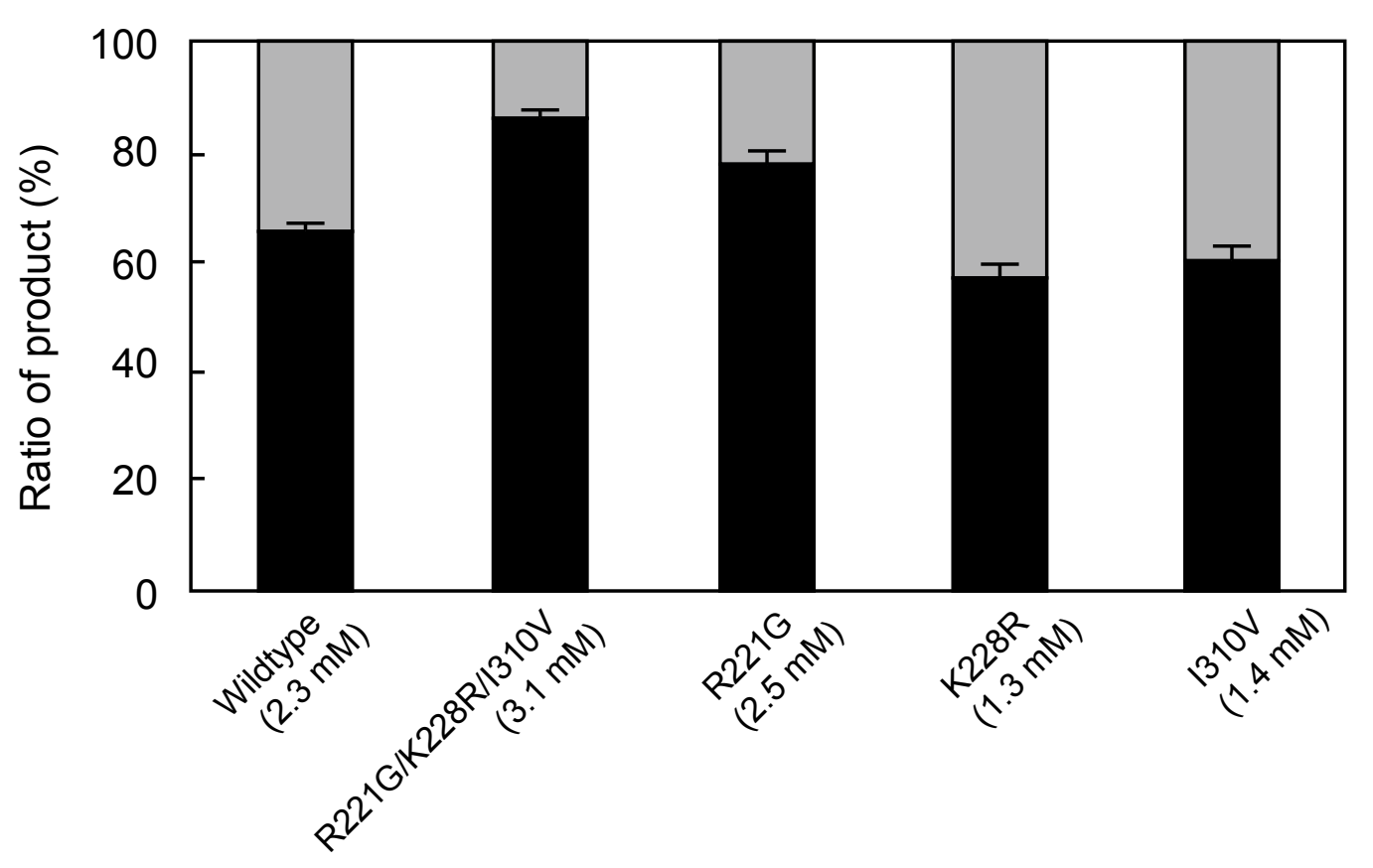

Morimoto et al., FIG. 2 


$\begin{array}{lccll}\text { Enzyme } & \begin{array}{c}\text { Cofactor } \\ \text { preference }\end{array} & & & \\ \text { TkME } & \text { NADP } & 209 & \text { VTVDRKGIIHEGR-EDLNPYKRE } & 230 \\ \text { ClME } & \text { NADP } & 342 & \text { WMVDSKGLIVKGR-ASLTPEKEH } & 364 \\ \text { Human } & \text { NADP } & 342 & \text { WLVDSKGLIVKGR-ASLTQEKEK } & 364 \\ \text { Rice } & \text { NADP } & 167 & \text { WLVDSKGLIVSSRKETLQHFKKP } & 189 \\ \text { Maize } & \text { NADP } & 389 & \text { WLVDSKGLIVDSRKGSLQPFKKP } & 411 \\ \text { E. coli } & \text { NAD } & 285 & \text { FMVDRFGLLTDKMPNLLPFQTKL } & 307 \\ \text { S. pombe } & \text { NAD } & 335 & \text { FMIDRCGLLLERHAKIATDGQKP } & 357\end{array}$

Morimoto et al., FIG. 3 

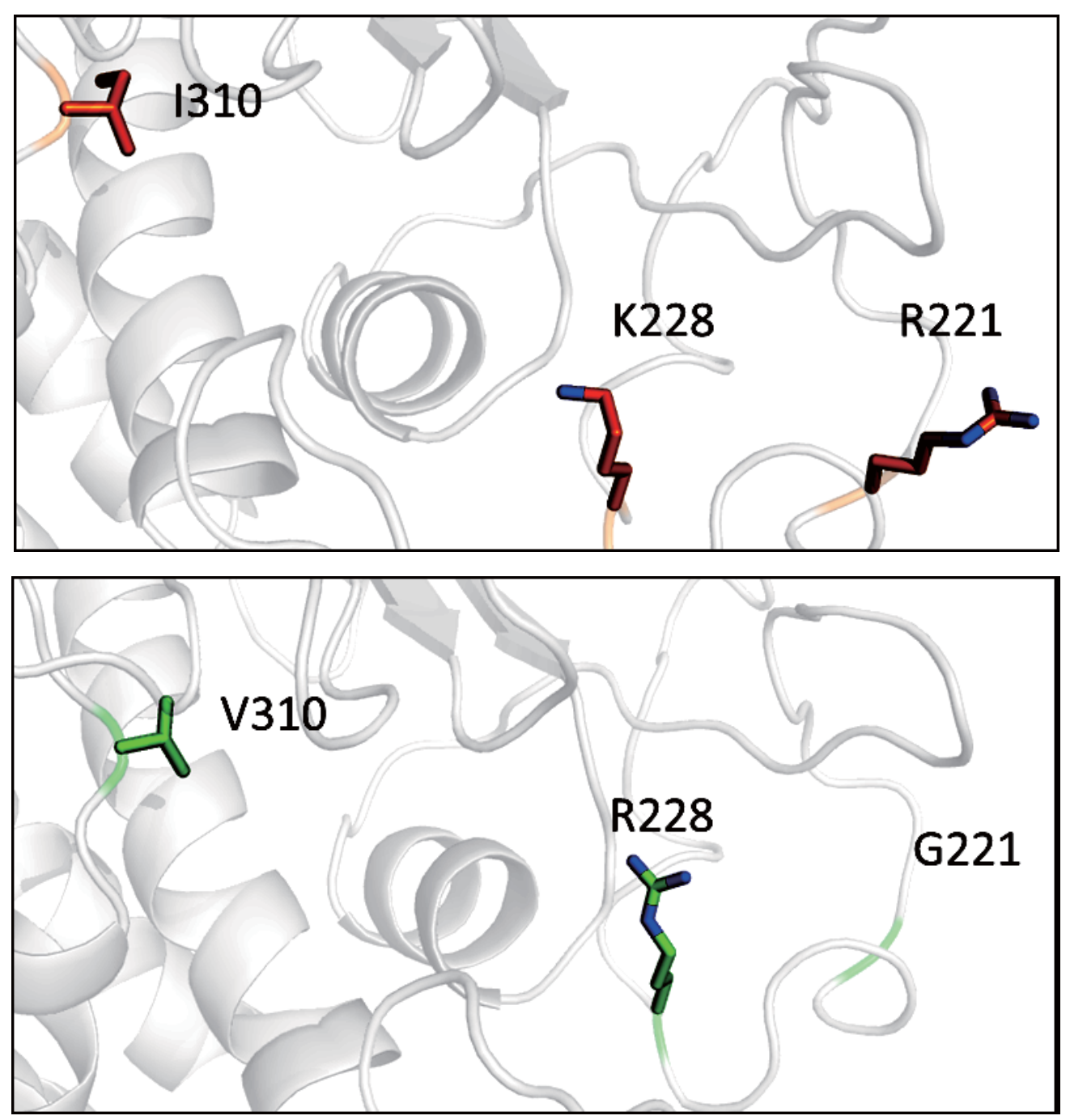

Morimoto et al., Fig. 4 